Aufklärung und praktische Beratung

Chemo: Die Zeit ohne

Haare überstehen

Die Alopezie als Nebenwirkung einer Chemotherapie wird von vielen Frauen als Alptraum empfunden. Um den Schock beim einsetzenden Haarausfall abzumildern, ist es wichtig, im Vorfeld so genau wie möglich über die Abläufe aufzuklären und den Betroffenen mit praktischen Tipps zur Seite zu stehen.

Trotz aller Fortschritte in der Behandlung toxischer Nebenwirkungen einer Chemotherapie bleibt der Haarausfall ein schwer zu lösendes Problem. In einer Studie von Nadège Carelle und Kollegen [1] rangierte der Haarverlust auf der Liste der Nebenwirkungen einer Chemotherapie an zweiter Stelle - noch vor der Fatigue. An erster Stelle stand die Belastung, die die Therapie für Familie oder Partner darstellt. Manche an Brustkrebs erkrankten Frauen würden sogar so weit gehen, weniger wirksame Therapien zu wählen oder sich gar nicht behandeln zu lassen, nur um ihre Haare zu behalten.

\section{Aufklärung hilft}

In verschiedenen Studien kommen die Forscher zu dem Schluss, dass die Alopezie besser toleriert wird, wenn im Vorfeld eine ausführliche Aufklärung stattgefunden hat. Durch das mit der Aufklärung verbundene bessere Verständnis der Patienten könnte emotionalem Stress, Ängsten und Depressionen zudem besser entgegengewirkt werden, so Hesketh und Kollegen [2].

\section{Wie kommt es zum Haarverlust?}

Vor Therapiebeginn sollten Ärzte die Patienten darüber aufklären, wie groß das Risiko bei ihrem Behandlungsregime ist, die Haare zu verlieren, und warum dies geschieht.

Zytostatika führen nicht nur bei Tumorzellen, sondern auch bei den Haarwurzelzellen zur Apoptose. Da die Therapie vor allem auf solche Zellen abzielt, die sich rasch teilen, sind die Zellen der Haarmatrix, die sich in der Wachstumsphase (anagene Phase) befinden, besonders stark betroffen.

\section{Wie geht der Haarausfall vonstatten?}

Die Alopezie beschränkt sich nicht nur auf den Kopf, auch Schamhaare, Achselhaare und Gesichtshaare fallen aus. In der Regel beginnt sie sieben bis zehn Tage nach Beginn der Chemotherapie. Am ausgeprägtesten ist die Alopezie innerhalb der ersten beiden Monate der Therapie und in den zwei bis drei Wochen nach Therapieende

Insgesamt dauert es in der Regel drei bis sechs Monate, bis der Kopf wieder vollständig von Haaren bedeckt ist. Nach einer Untersuchung von Diane Batchelor [3] sehen die nachwachsenden Haare bei etwa $65 \%$ der Betroffenen anders aus als vor der Chemotherapie. Bei einigen sind die Haare danach grau, sie können aber auch dunkler werden. Etwa bei jeder dritten Patientin veränderte sich in einer Studie die Struktur der Haare. Oft sind sie dann gewellt; sie können aber auch dünner sein oder sich rauer anfühlen.

\section{Tipps für den Umgang mit dem Haarverlust}

Wenn die Patienten diese vorhersehbaren Abläufe kennen, können sie besser damit umgehen. Um Haarbüschel im Bett zu vermeiden, kann z. B. ein Haarnetz getragen werden. Kämme mit großem Zinkenabstand oder weiche Bürsten sind zu bevorzugen, damit nicht unnötig Haare ausgerissen werden.

Tägliches Haarewaschen sollten die Patienten vermeiden. Renate Haidinger, selbst im Jahr 2000 an Brustkrebs erkrankt und heute 1. Vorsitzende des Vereins „Brustkrebs“, empfiehlt, die Haare in jedem Fall bei den ersten Anzeichen des Ausfalls komplett abzurasieren: „Sonst

\section{haben Sie die Haare überall, am Boden, im Bett, im Essen." Diese für die Patien- tin schwer zu verkraftende Phase sollte so kurz wie möglich andauern. \\ Zu den am häufigsten genutzten Strate- gien, um den Verlust des Haupthaars zu kompensieren, gehört die Perücke. Exper- ten raten, diese auszusuchen, solange die eigenen Haare noch vorhanden sind; so kann man das Haarteil in Farbe und Tex- tur am besten an den natürlichen Zustand anpassen. Für Frauen mit langen Haaren besteht die Möglichkeit, sich eine Perücke aus Eigenhaar anfertigen zu lassen. Hai- dinger empfiehlt allerdings eine Kunst- haarperücke: Diese sei wesentlich pflege- leichter. Kopftücher oder Hüte kommen in solchen Fällen als Alternativen infrage. \\ Der haarlose Kopf bedarf besonderer Schutzmaßnahmen. Empfehlenswert ist es, zu starke Sonnenexposition zu ver- meiden, Sonnenschutz zu verwenden und den Kopf vor Kälte zu schützen. Hautir- ritationen lässt sich mit Cremes oder Ba- byöl vorbeugen. Oft vernachlässigt wird der Augenschutz: Durch fehlende Wim- pern ist das Auge viel stärker dem Licht ausgesetzt als vorher; daher wird den $\mathrm{Pa}$ - tienten geraten, an hellen Tagen eine Son- nenbrille zu tragen. \\ Tipps für die Praxis \\ Der Verein „Brustkrebs“ hat viele nützli- che Tipps in der Broschüre „Brustkrebs und Haarverlust" zusammengestellt. Sie kann kostenlos über info@brustkrebs- deutschland.de angefordert werden. Da- rin enthalten sind Ratschläge für die Pfle- ge von Haaren und Kopfhaut, Bindetech- niken für Kopftücher und Schminktipps. Zudem können sich Patientinnen unter der Telefonhotline 0800 / 0117112 kos- tenlos beraten lassen. \\ (Elke Oberhofer) \\ 1. Carelle N et al. Cancer. 2002;95(1):155-63}

2. Hesketh PJ et al. Support Care Cancer.

2004;12(8):543-9

3. Batchelor D. Eur J Cancer Care (Engl).

2001;10(3):147-63 